UNITED STATES DEPARTMENT OF THE INTERIOR

GEOLOGICAL SURVEY

\title{
Map and table describing fossil collections and related samples in the Ketchikan and Prince \\ Rupert quadrangles, southeastern Alaska
}

\section{by}

Henry C. Berg and Edwin L. Cruz

U.S. Geoiogical Survey

Open-File Report 82-1088

This report is preliminary and has

not been edited or reviewed for conformity with Geological Survey standards and nomenclature

Anchorage, Alaska

1982 
Map and table describing fossil collections and related samples in the Ketchikan and Prince Rupert quadrangles, southeastern Alaska

by

Henry C. Berg and Edwin L. Cruz

INTRODUCTION

This report consists of a map and companion table describing (a) fossil collections that have been made in the Ketchikan and Prince Rupert quadrangles east of Clarence Strait, and (b) samples collected for fossil (mainily conodont) determinations, whether or not they actually yielded conodonts or other fossils. The purpose of the report is to summarize in a single publication information about fossils gathered in the area by numerous workers since before 1900; some of this information has not previously been publically available. The report supplements published geologic maps of Annette and Gravina Islands (Berg, 1972, 1973) and of the Ketchikan and Prince Rupert quadrangles (Berg and others, 1978), and is one of a series of reports stemming from geological and mineral resource investigations of the area conducted under the Alaska Mineral Resource Assessment Program (AMRAP) (Berg, 1982).

Sources of information compiled for this report mainly are books and maps published by the U.S. Geological Survey (USGS). These are cited in the table and are listed at the end of this introduction. In most cases, the cited work contains additional information about the geology, stratigraphy, or fauna at the site. Sources of unpublished data include formal and informal reports by USGS paleontologists describing some of the fossil collections, and notebooks and maps of USGS workers conducting geological and mineral resource studies in the area.

The map shows the sites of approximately 150 fossil localities plotted at two topographic map scales. The larger scale map covers the Gravina-AnnetteHotspur Islands area, which contains most of the fossil occurrences known in the Ketchikan and Prince Rupert quadrangles. The other map encompasses both quadrangles and shows the sites of the dozen-odd fossil occurrences known outside the Gravina-Annette-Hotspur area. This map also is a generalized geologic map showing the distribution of major rock units in the quadrangles, and is the reference for the map units listed in the first column of the table. Data for the geologic map are generalized from several USGS publications that describe the geology in more detail (Berg, 1972, 1973, 1982; Berg and others, 1977, 1978).

The table summarizes information available about each fossil occurrence or related sample and includes, insofar as is known: geologic map unit; lithology; collector, year, and (when appropriate) USGS Museum collection number; names and ages of fossils; name of the person who identified the iossils; and citations of published references or other sources of data.

Presentation of data: Some of the most complete fossil collections and related descriptions were made by early workers when many of the sites were accessible by mining or logging roads. The table thus lists fossil collections in approximate chronological order, starting with the earliest collections reported in the area. On the other hand, lack of adequate maps prevented these early workers from recording the exact locations of their collections, and many of their sites are only approximately located on the maps in this report. More than one number at a site indicates that it was examined more than once, and these numbers are also in approximate chronological order. 
Presentation of data: Some of the most complete fossil collections and related descriptions were made by early workers when many of the sites were accessible by mining or logging roads. The table thus lists fossil collections in approximate chronological order, starting with the earliest collections reported in the area. Lack of adequate maps prevented many of these early workers from recording the exact locations of their collections, and many of their sites are only approximately located on the maps in this report. Such approximately located sites are indicated by the symbol (A) in the second column of the table. More than one number at a site indicates that it was examined more than once, and these numbers are also in approximate chronological order.

Fossil identification and nomenclature: Except for omitting customary italics for generic and specific names, this report presents names of fossils identified in the collections exactly as originally published or otherwise reported. Subsequent taxonomic revisions may have invalidated some reported names.

Lithology: To conserve space, abbreviations are used in the left column of the table to summarize the lithology at each occurrence. A key to these lithologic abbreviations, together with examples of how to use them, follows.

\section{KEY TO LITHOLOGIC ABBREVIATIONS USED IN THE LEFT COLUMN OF THE TABLE}

ar: argillite, argillaceous
cc: calcareous
cg: conglomerate
cl: clast
cn: concretion, concretionary
dt: dolomite, dolomitic
gr: graphitic
gt: grit, gritty
gw: graywacke
li: lithographic

1s: limestone

mb: marble

ph: phyllite, phyllitic

py: pyrite, pyritic

sc: schist, schistose

sl: slate, slaty

ss: sandstone, sandy

st: siltstone, silty

tf: tuff, tuffaceous

Examples of how abbreviations are used:

(1) 1s: fossils occur in limestone

(2) ph gw: fossils occur in phyllitic graywacke

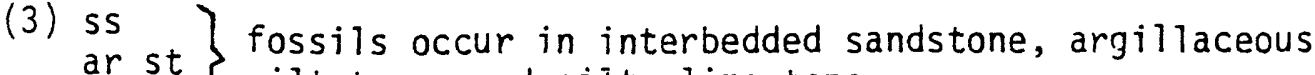
$\left.\begin{array}{l}\text { ar st } \\ \text { st } 1 \mathrm{~s}\end{array}\right\}$ siltstone, and silty limestone 


\section{REFERENCES CITED}

Berg, H.C., 1972, Geologic map of Annette Island, Alaska: U.S. Geological Survey Miscellaneous Geologic Investigations Map I-684, scale 1:63,360.

1973, Geology of Gravina Island, Alaska: U.S. Geological Survey Bulletin 1373, $41 \mathrm{p}$.

1982, The Alaska Mineral Resource Assessment Program: Guide to information about the geology and mineral resources of the Ketchikan and Prince Rupert quadrangles, southeastern Alaska: U.S. Geological Survey Circular 855, $24 \mathrm{p}$.

Berg, H.C., Elliott, R.L., Smith, J.G., and Koch, R.D., 1978, Geologic map of the Ketchikan and Prince Rupert quadrangles, Alaska: U.S. Geological Survey Open-File Report 78-73A, scale 1:250,000, 1 sheet.

Berg, H.C., Elliott, R.L., Smith, J.G., Pitman, T.L., and Kimball, A.L., 1977, Mineral resources of the Granite Fiords wilderness study area, Alaska: U.S. Geological Survey Bulletin 1403, 151 p.

Buddington, A.F., and Chapin, T., 1929, Geology and mineral deposits of southeastern Alaska: U.S. Geological Survey Bulletin 800,398 p.

Chapin, Theodore, 1918, The structure and stratigraphy of Gravina and Revillagigedo Islands, Alaska: U.S. Geological Survey Professional Paper 120-D, p. 83-100.

Martin, G.C., 1926, The Mesozoic stratigraphy of Alaska: U.S. Geological Survey Bulletin 776, 493 p.

Silberling, N.J., Wardlaw, B.R., and Berg, H.C., 1982, New paleontologic age determinations from the Taku terrane, Ketchikan area, southeastern Alaska, in Coonrad, W.L., ed., The United States Geological Survey in Alaska: Accomplishments during 1980: U.S. Geological Survey Circular 844 , p. $117-119$.

Smith, P.S., 1915, Notes on the geology of Gravina Island, Alaska, in Shorter contributions to general geology 1915: U.S. Geological Survey Professional Paper 95, p. 97-105.

Wright, F.E., and Wright, C.W., 1908, The Ketchikan and Wrangell mining districts, Alaska: U.S. Geological Survey Bulletin 347, 203 p. 


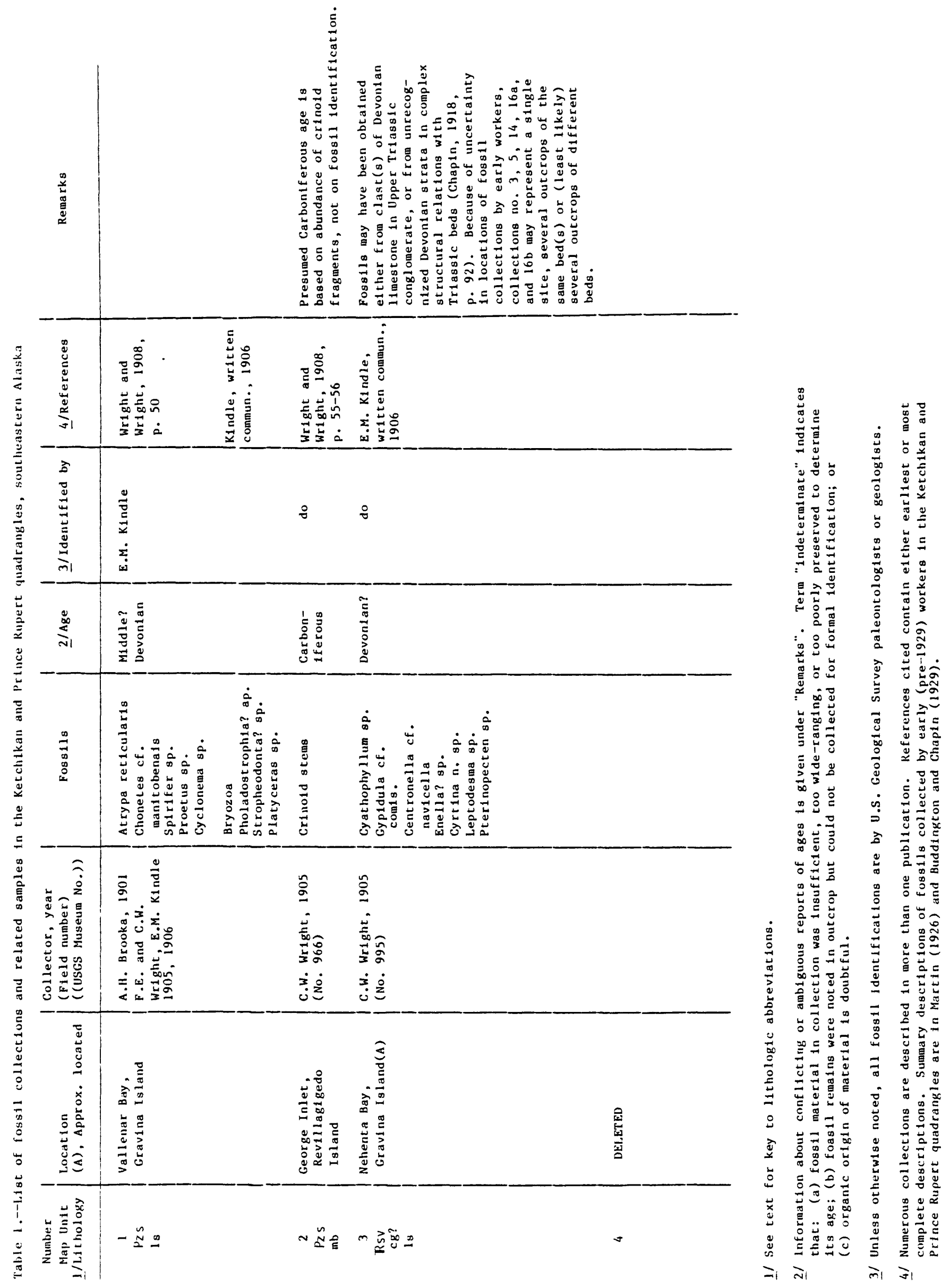




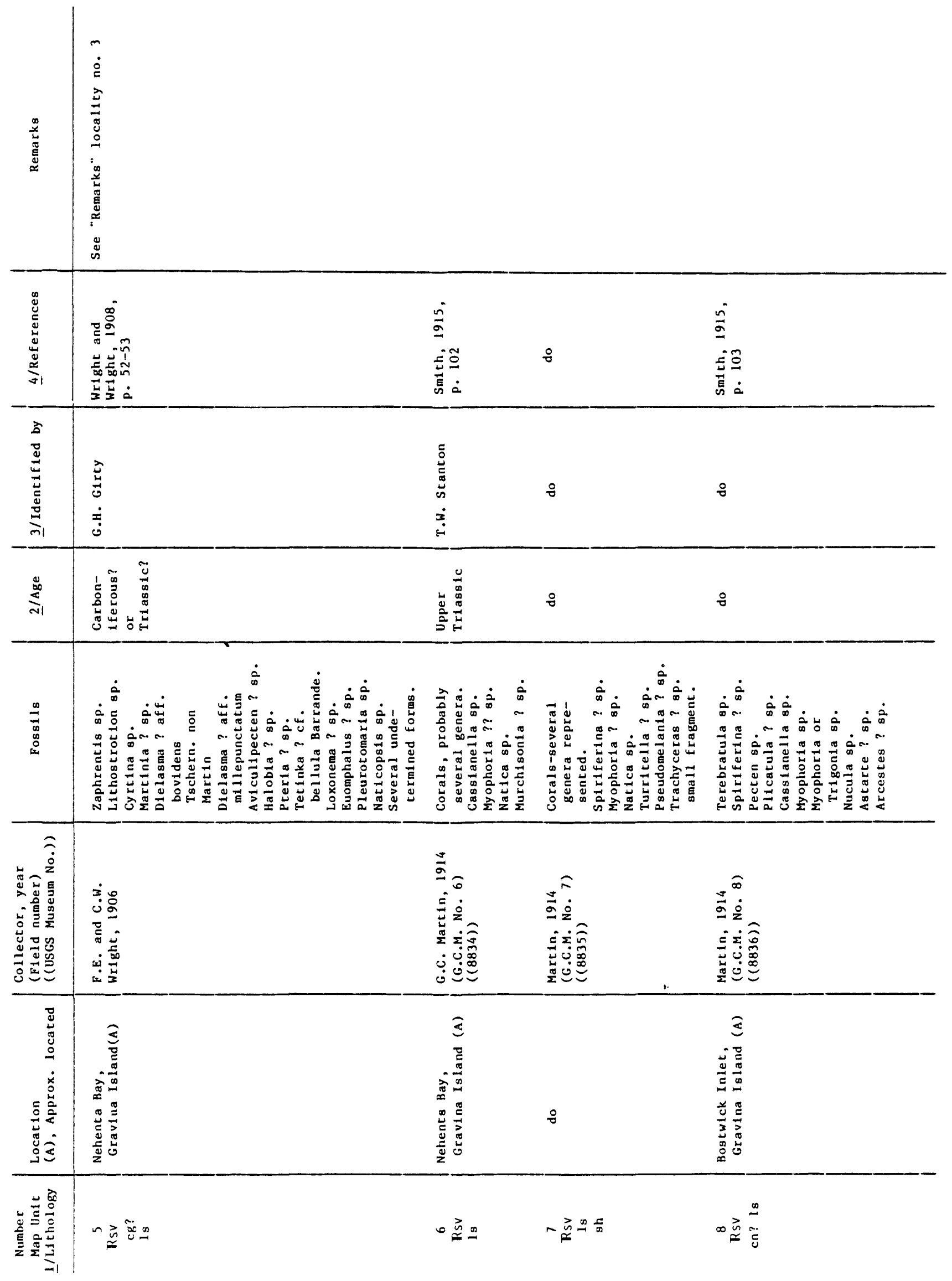




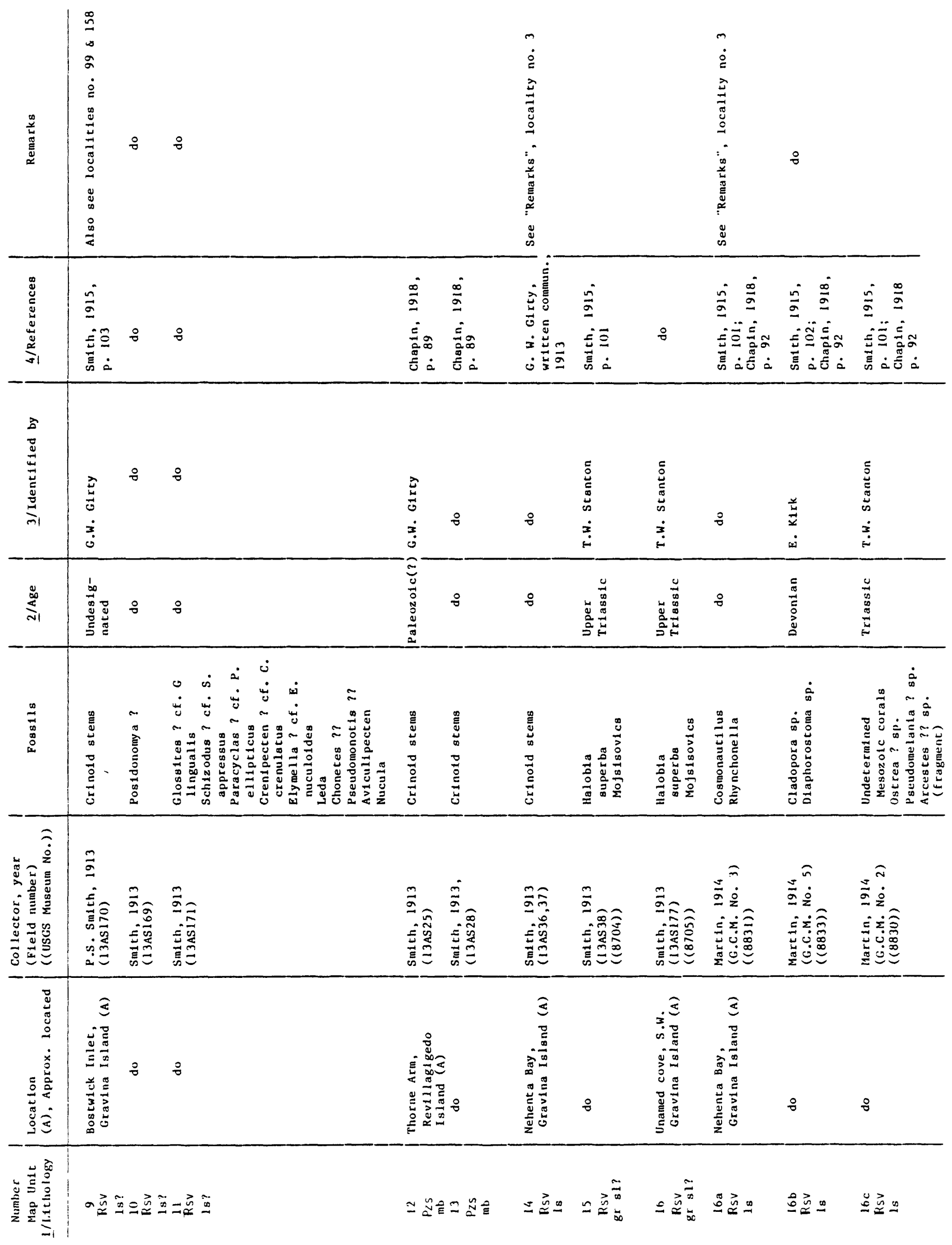




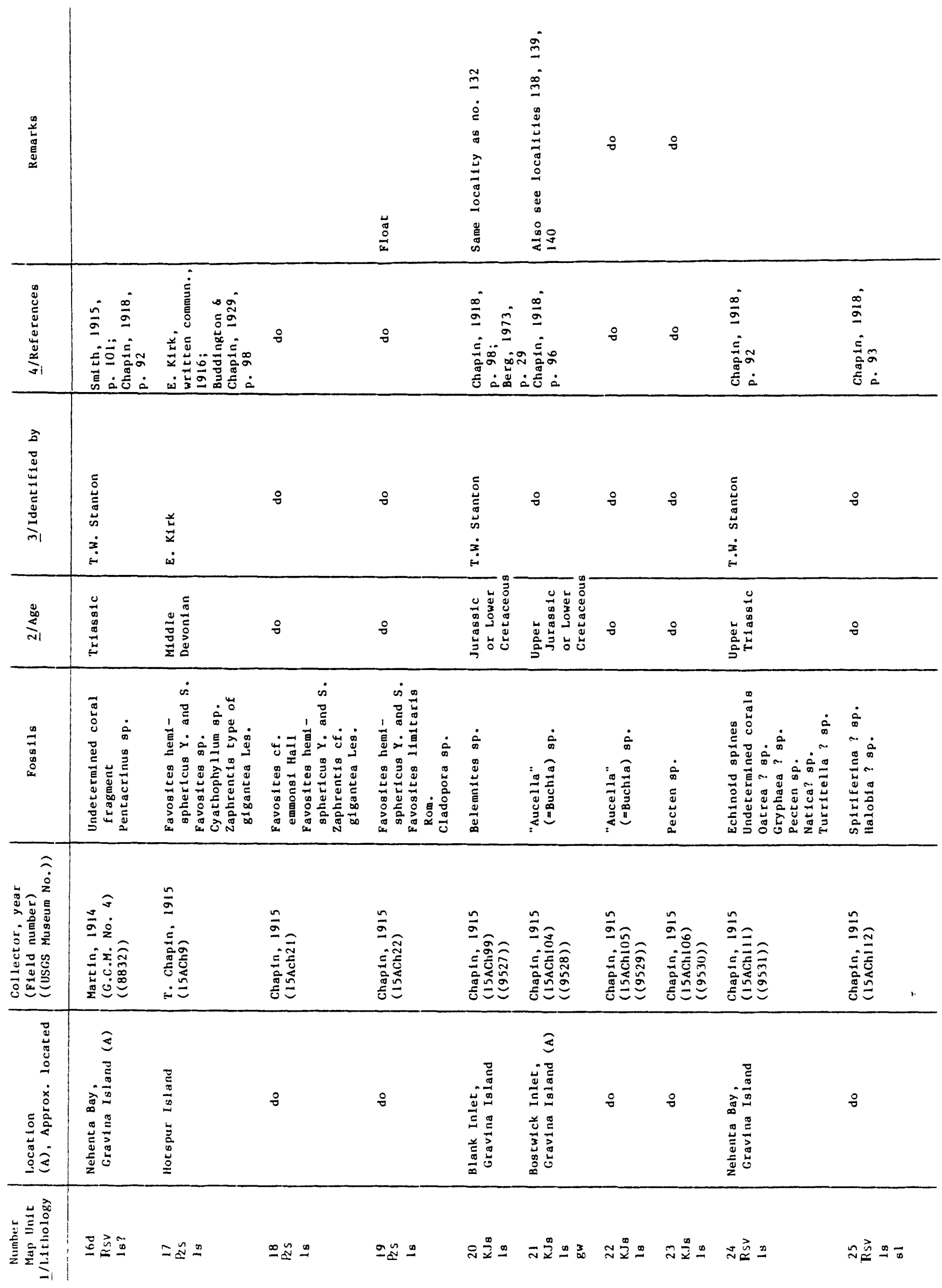




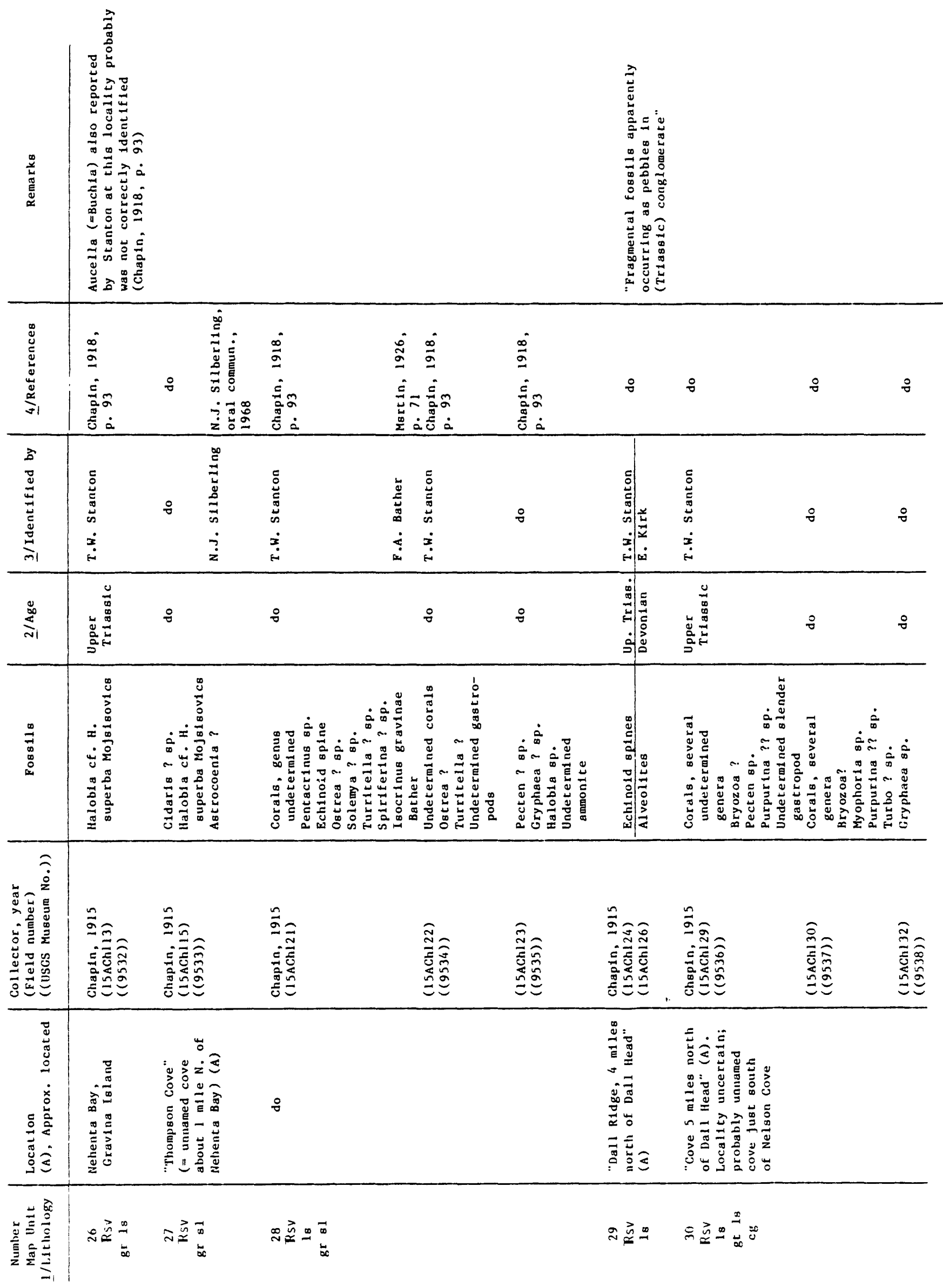




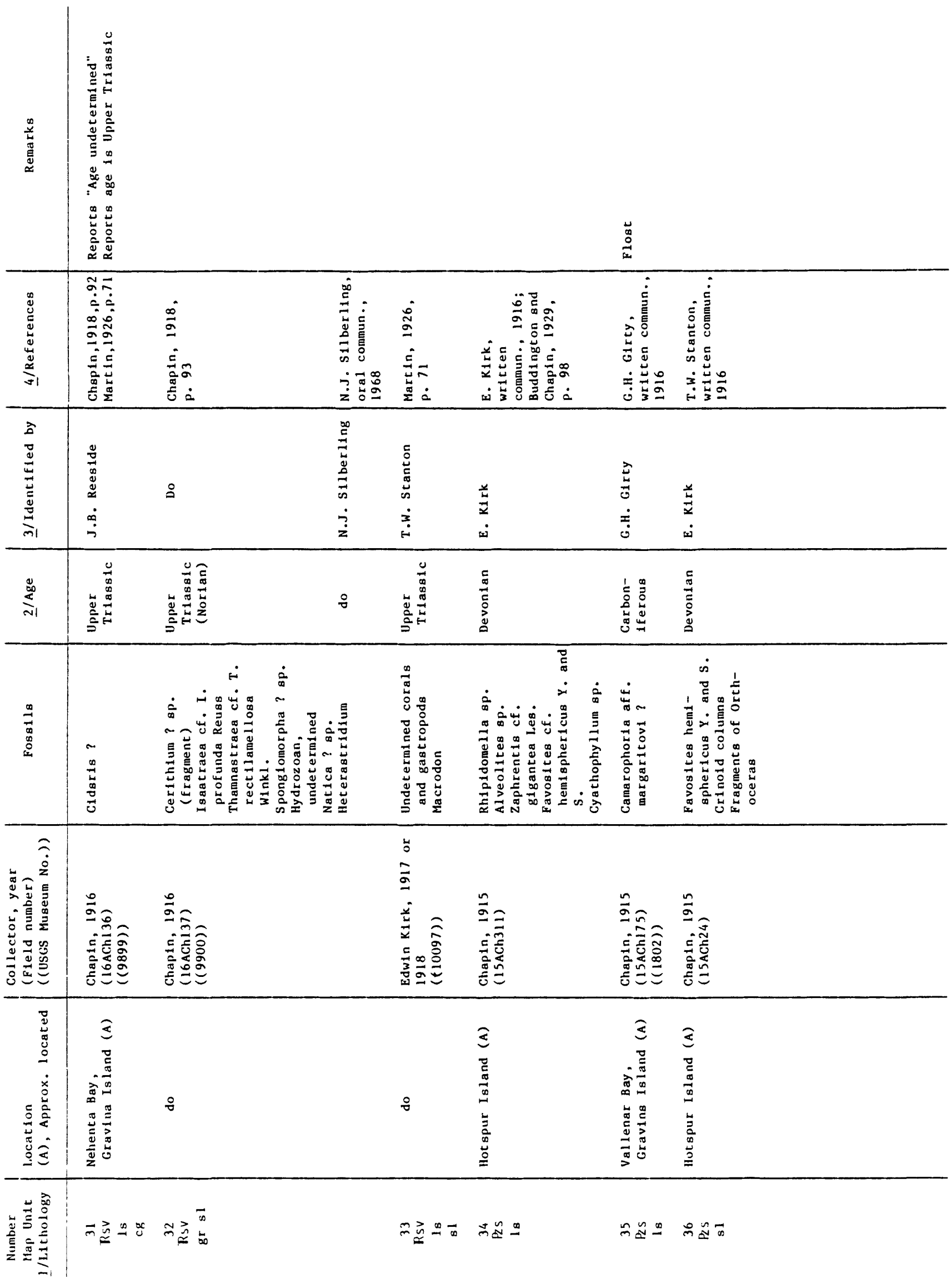




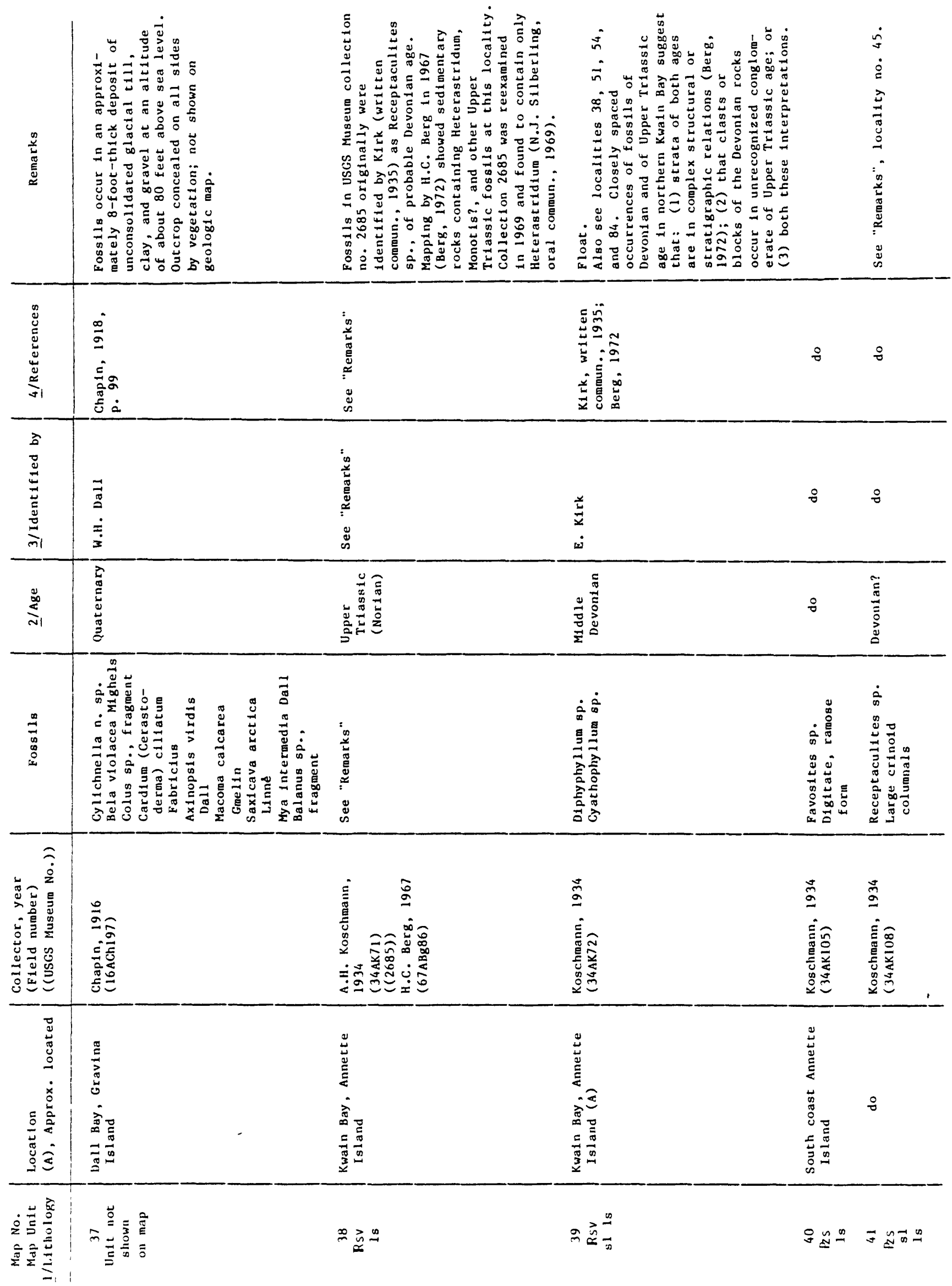




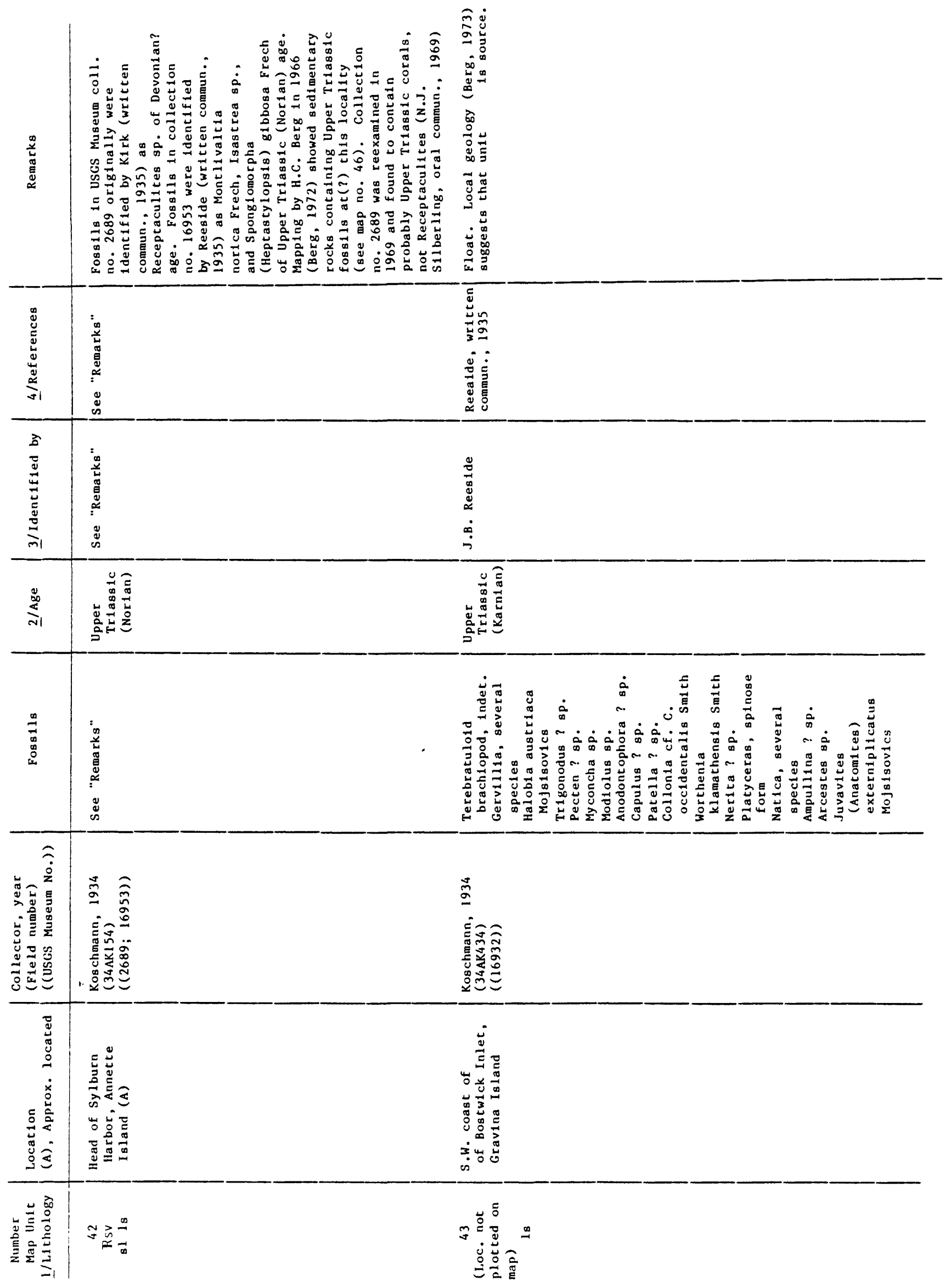




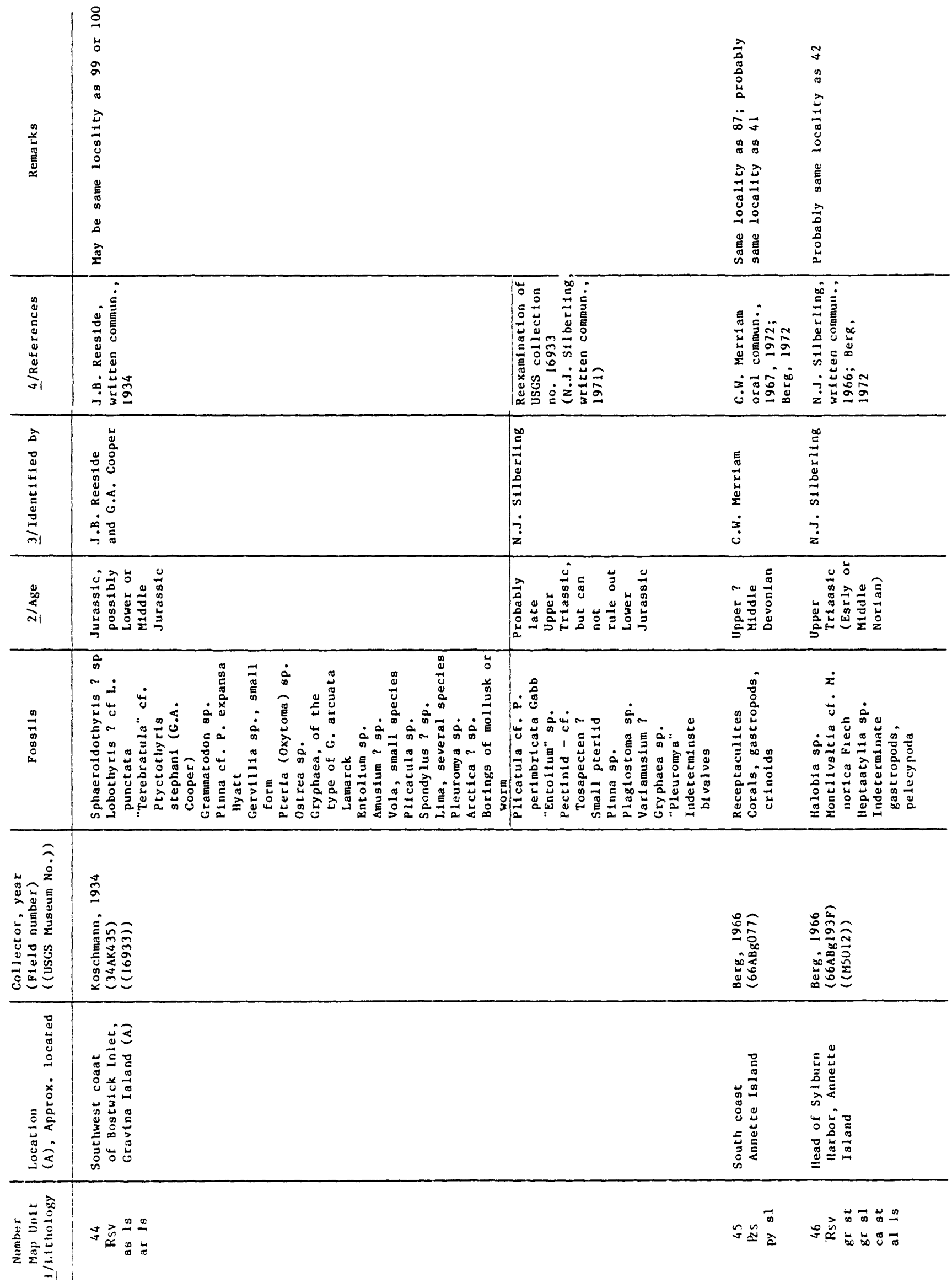




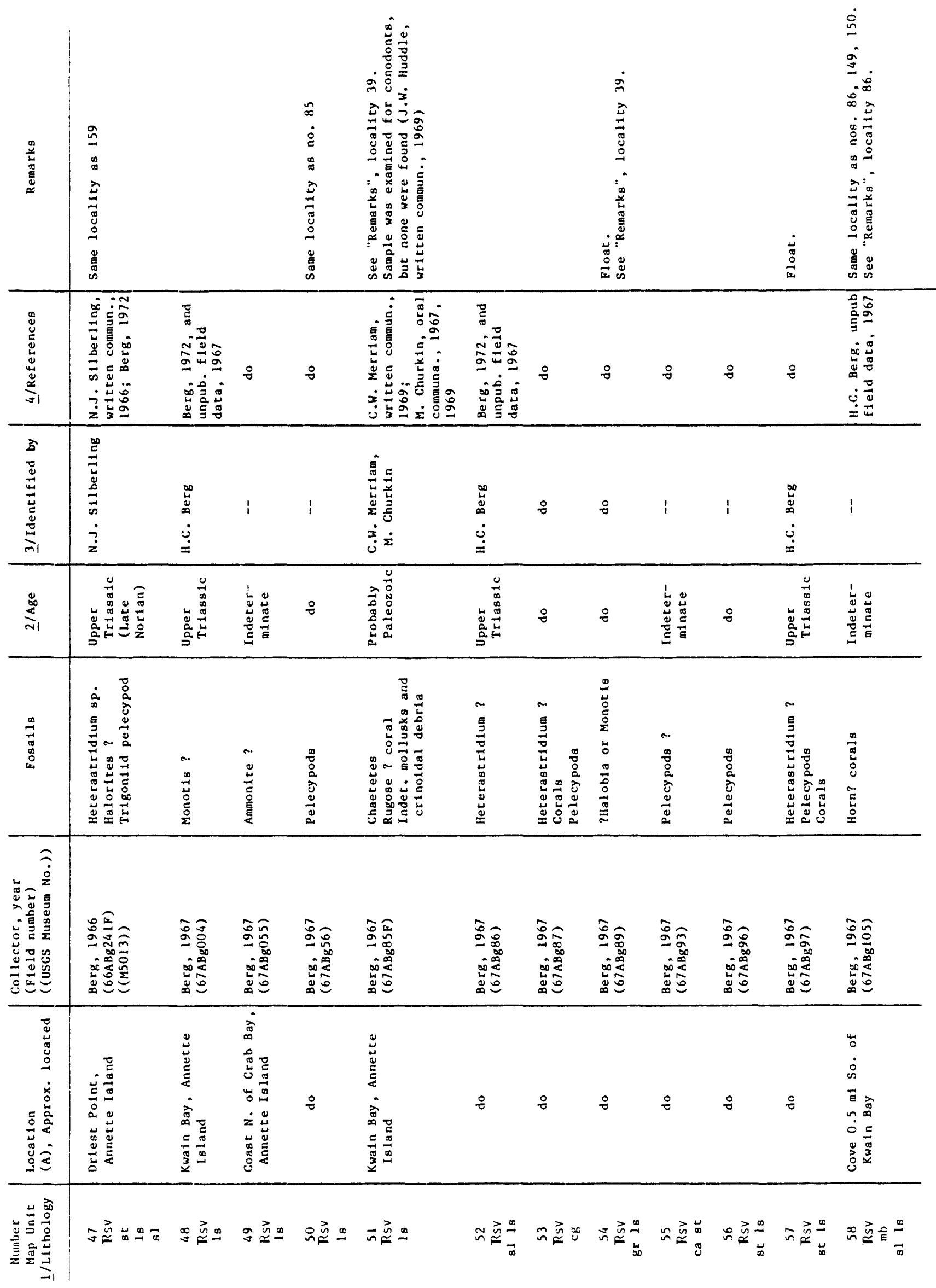




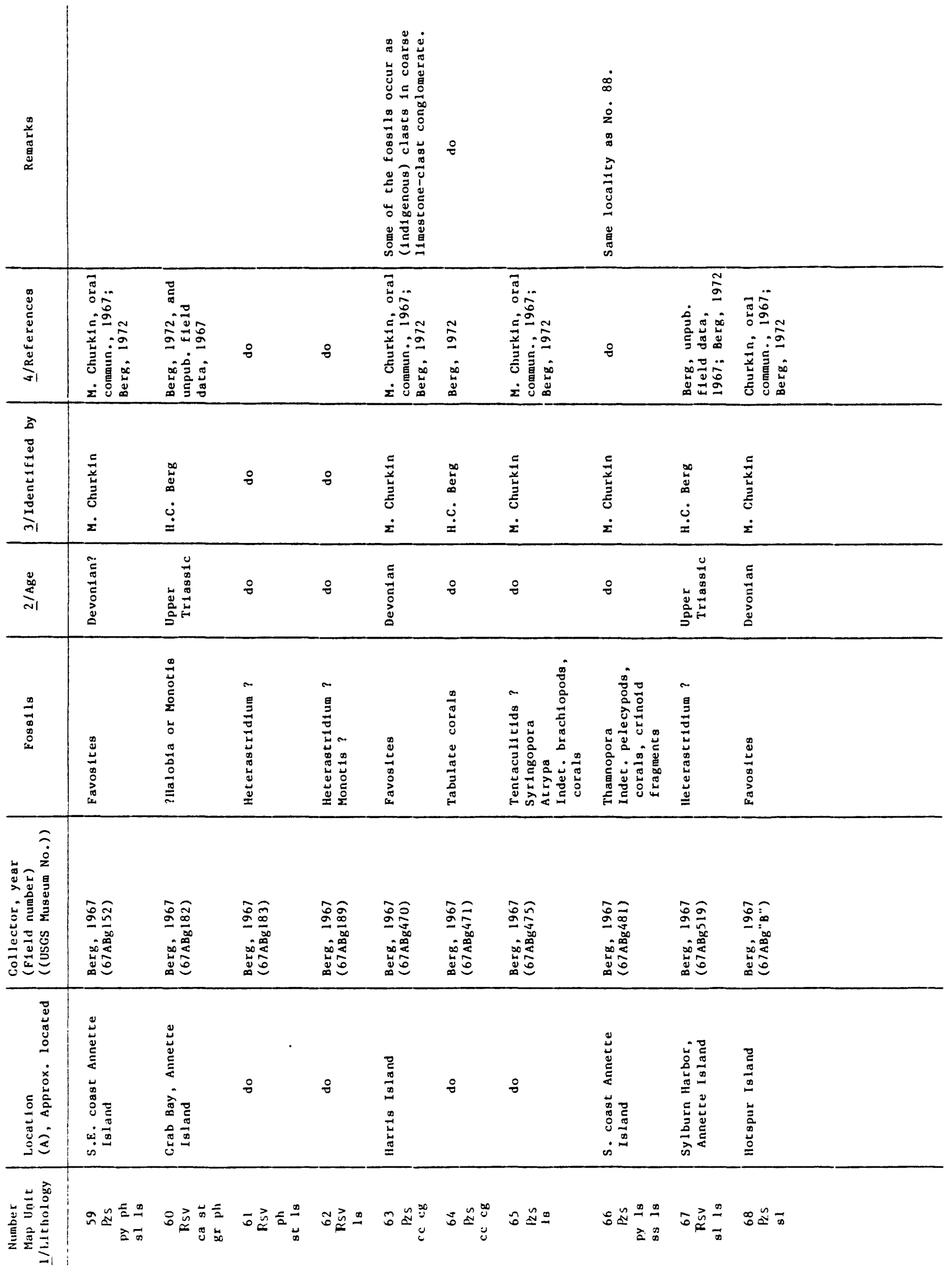




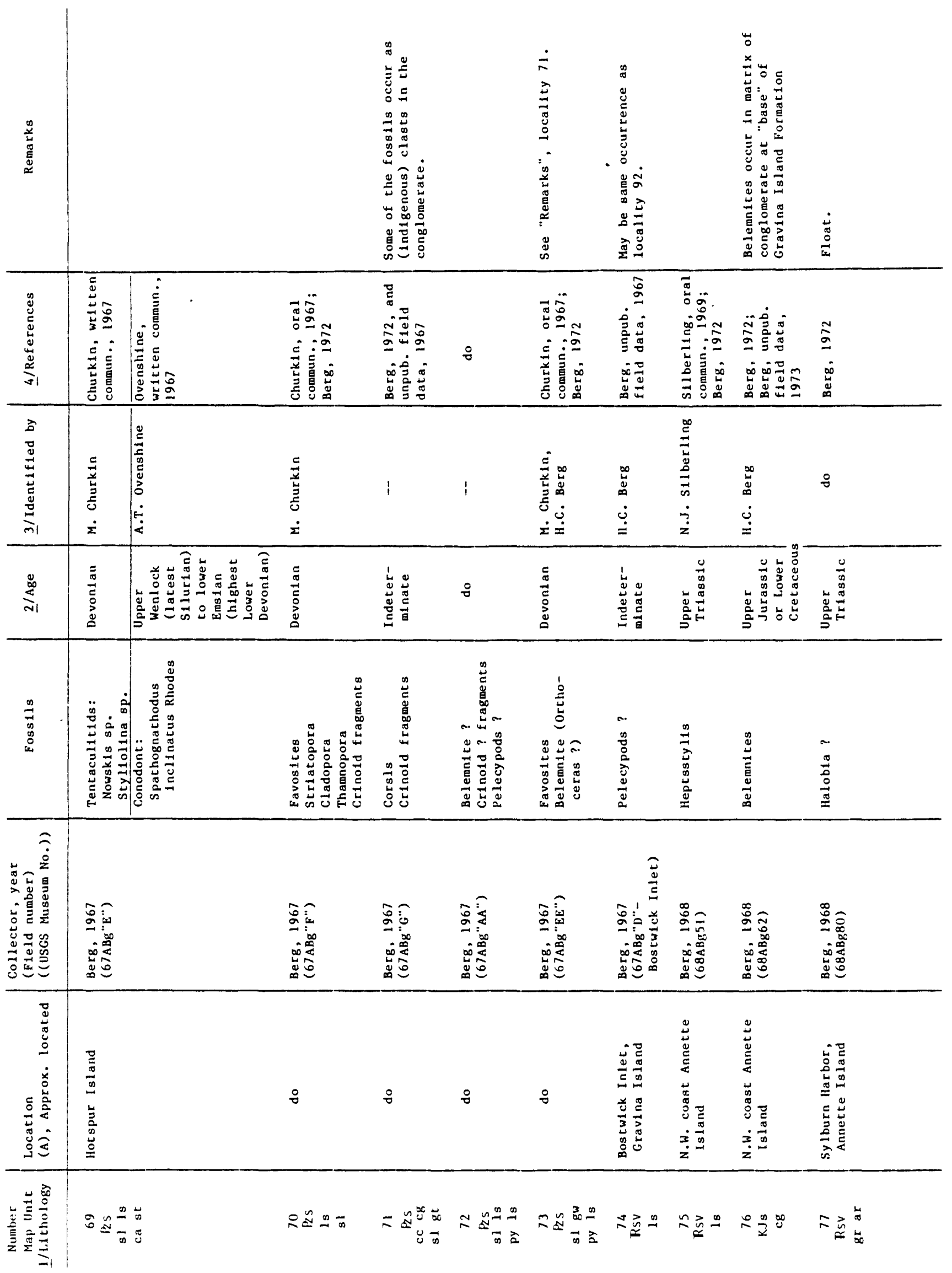



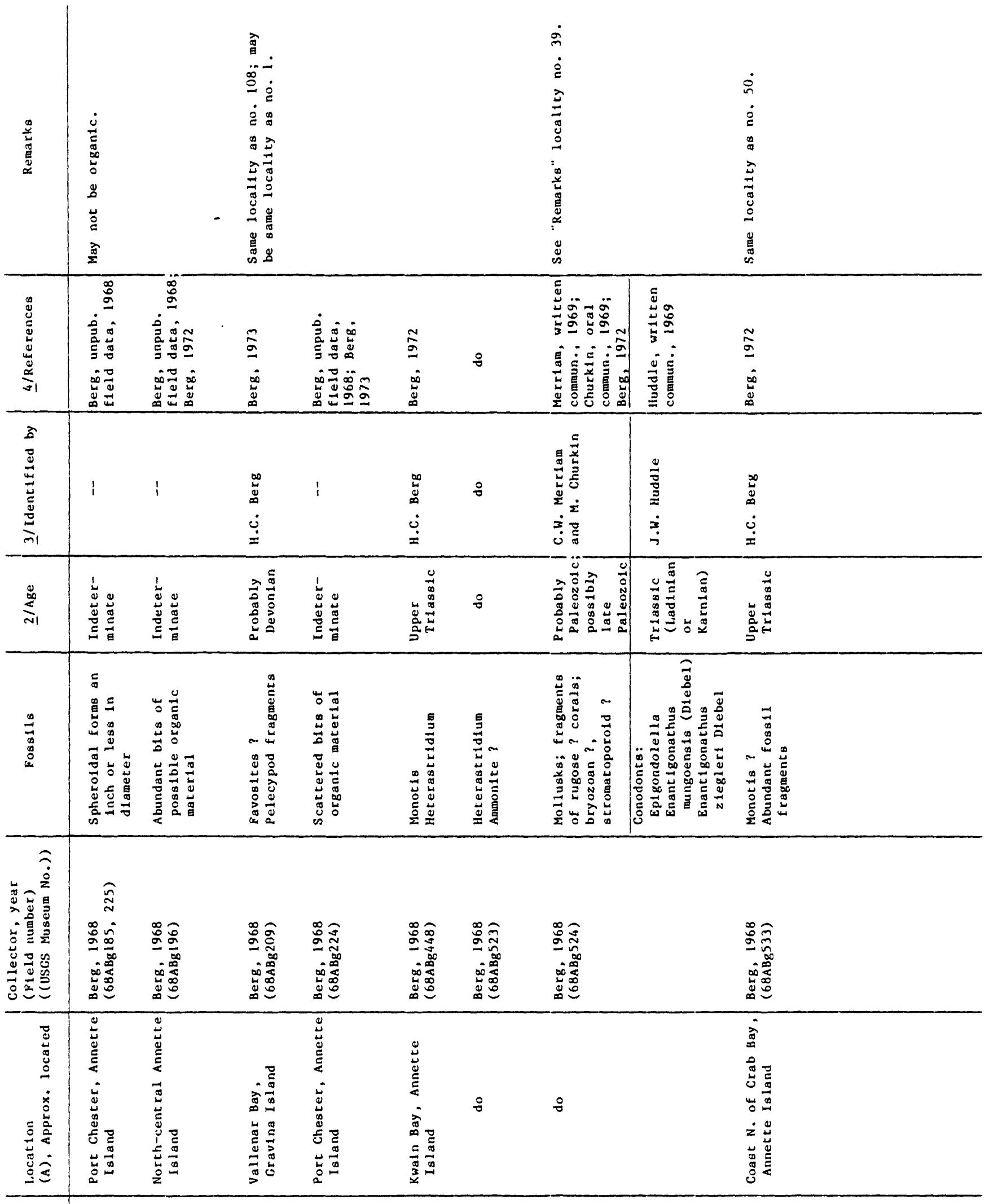

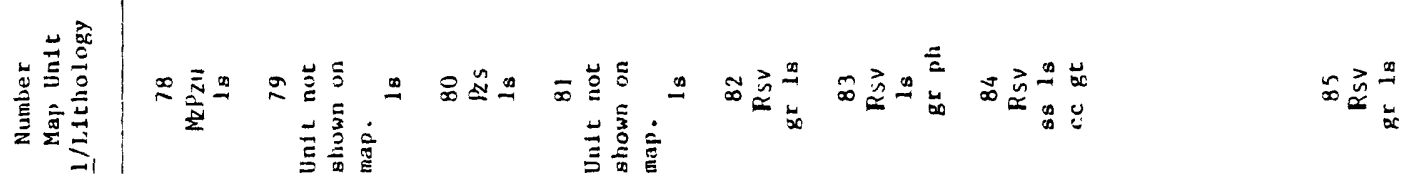




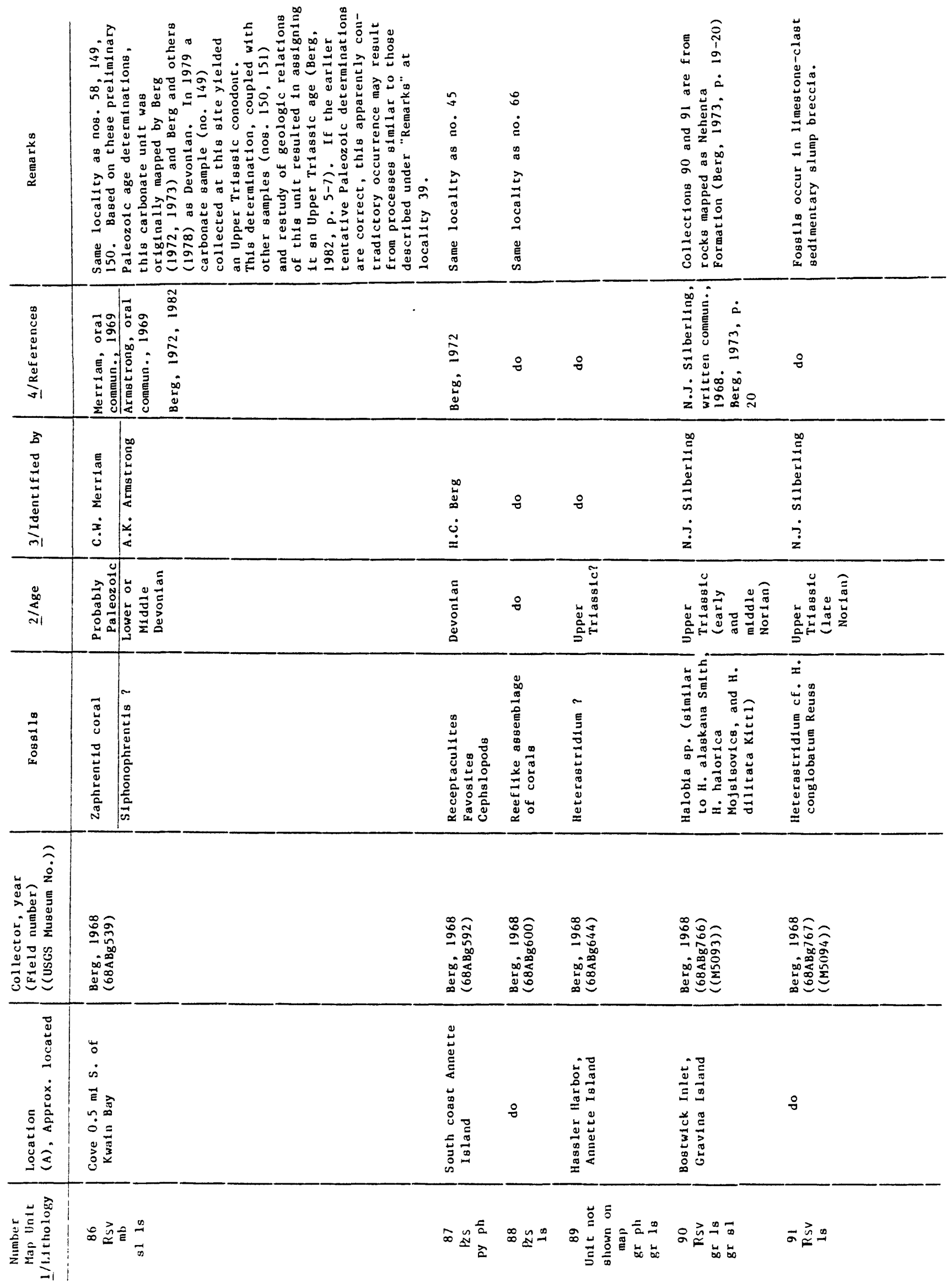




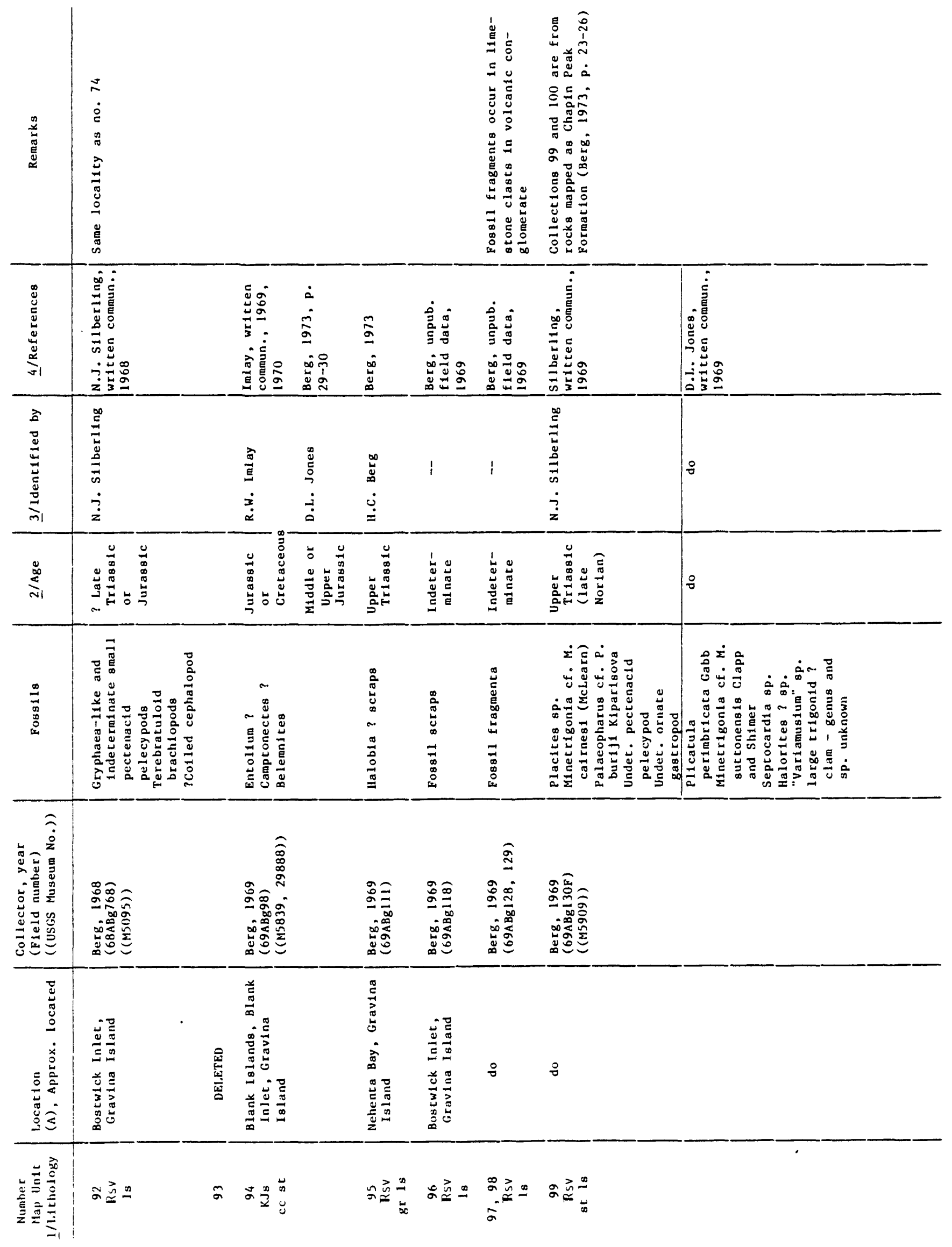




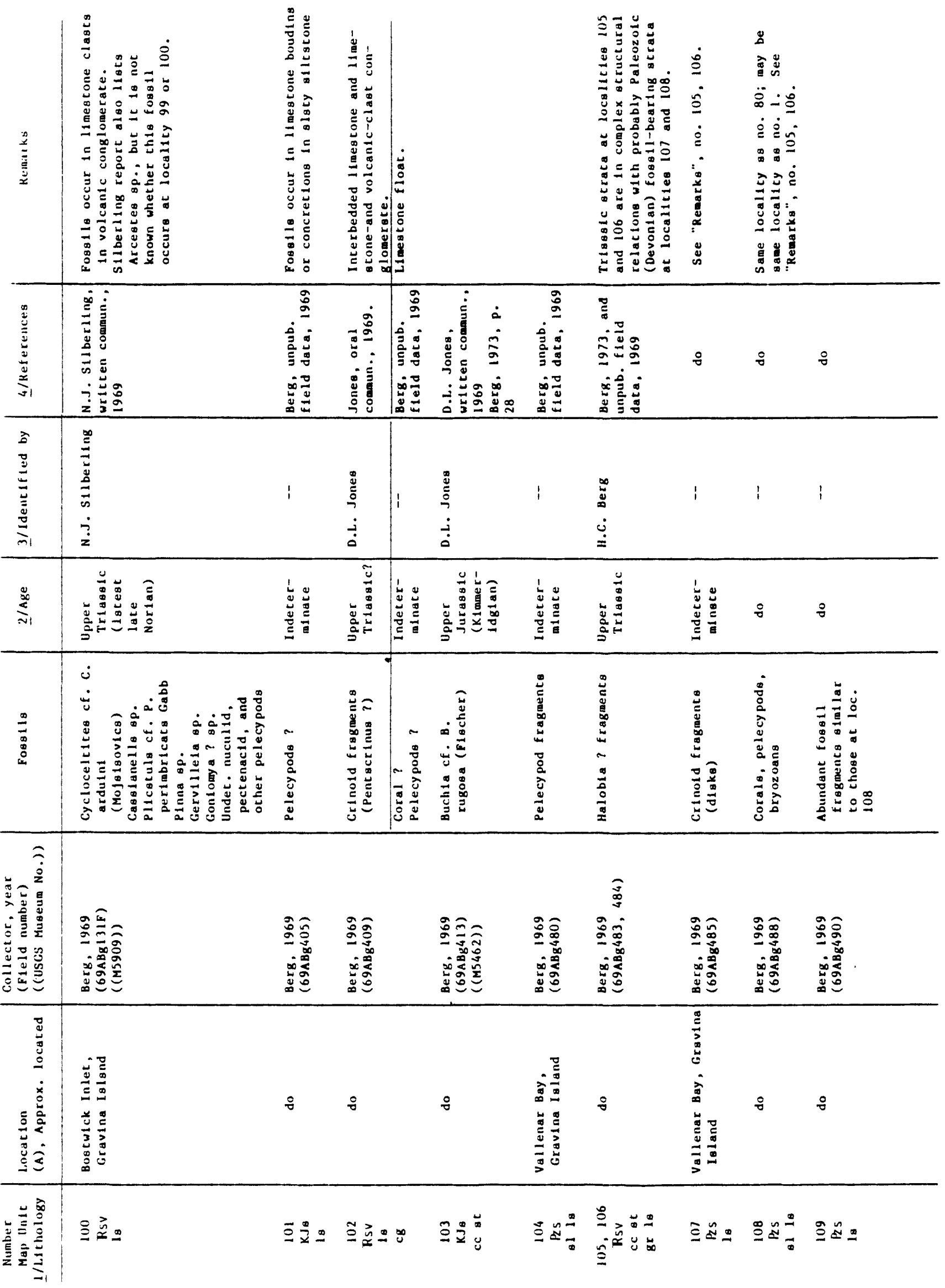




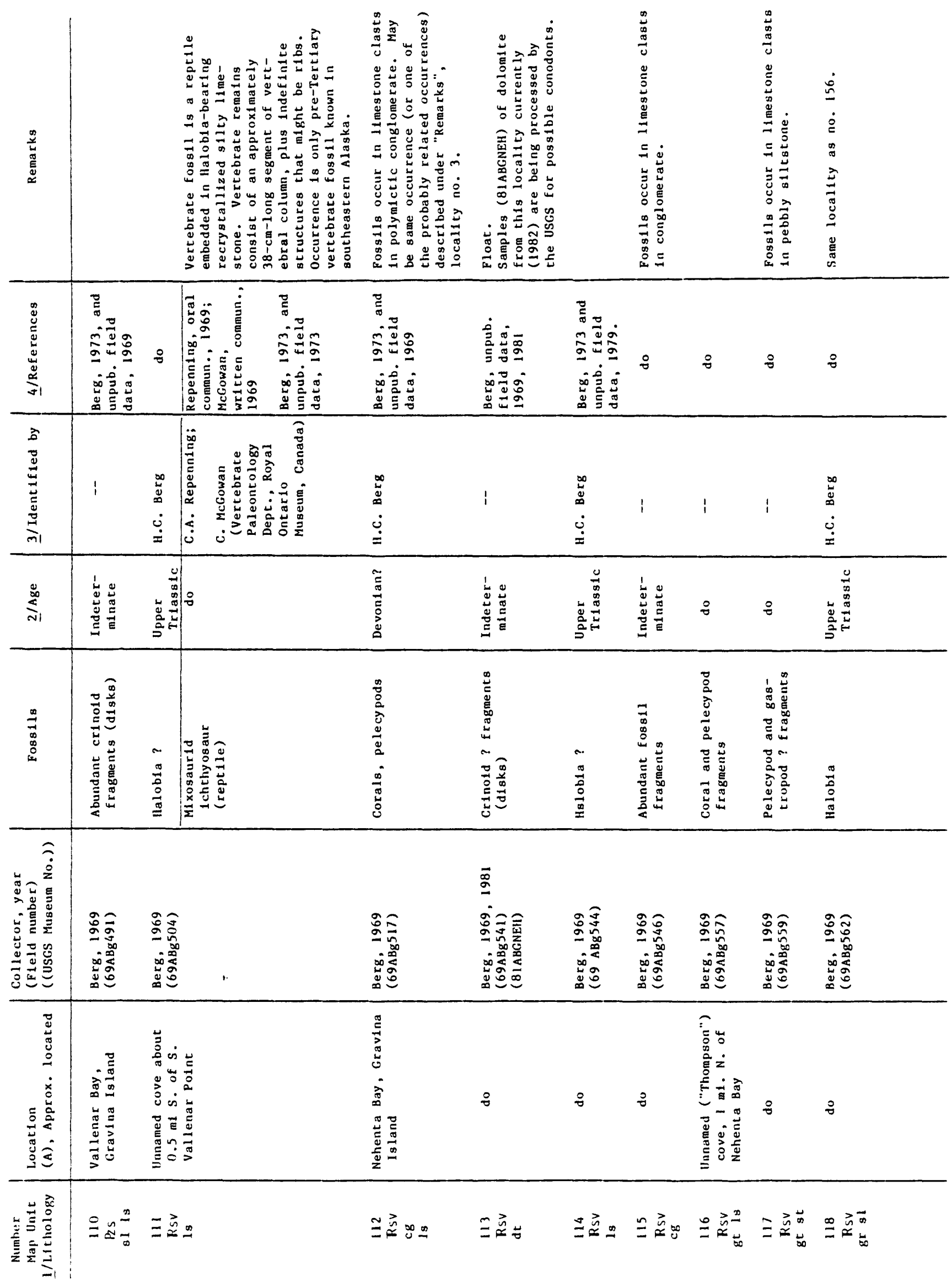




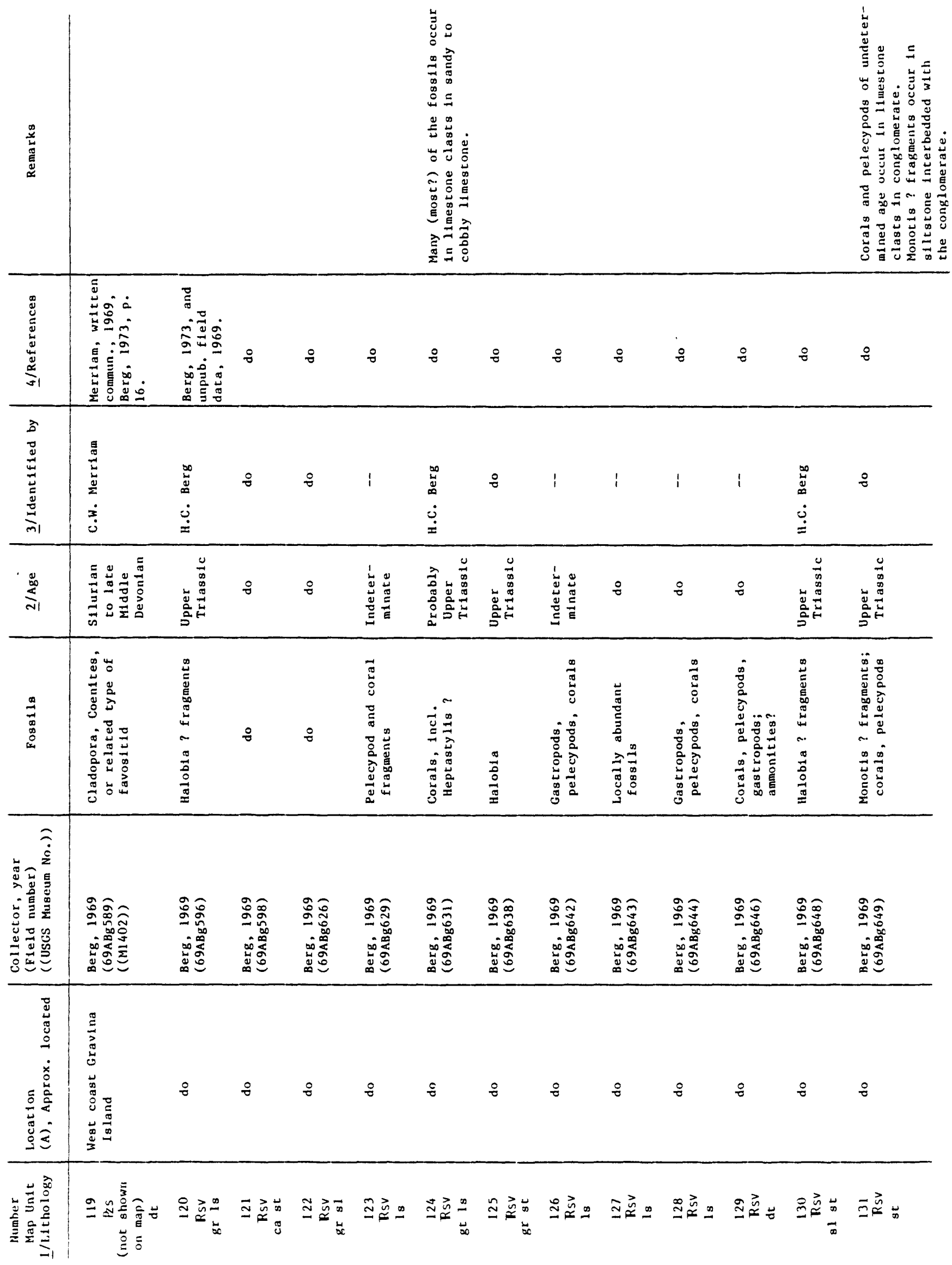




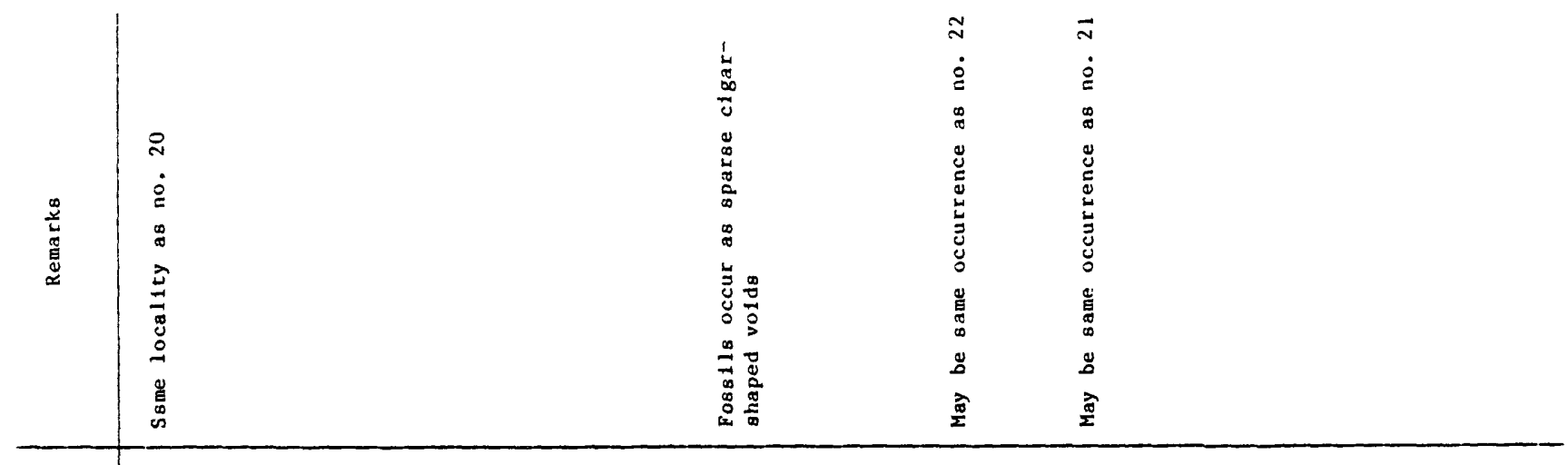

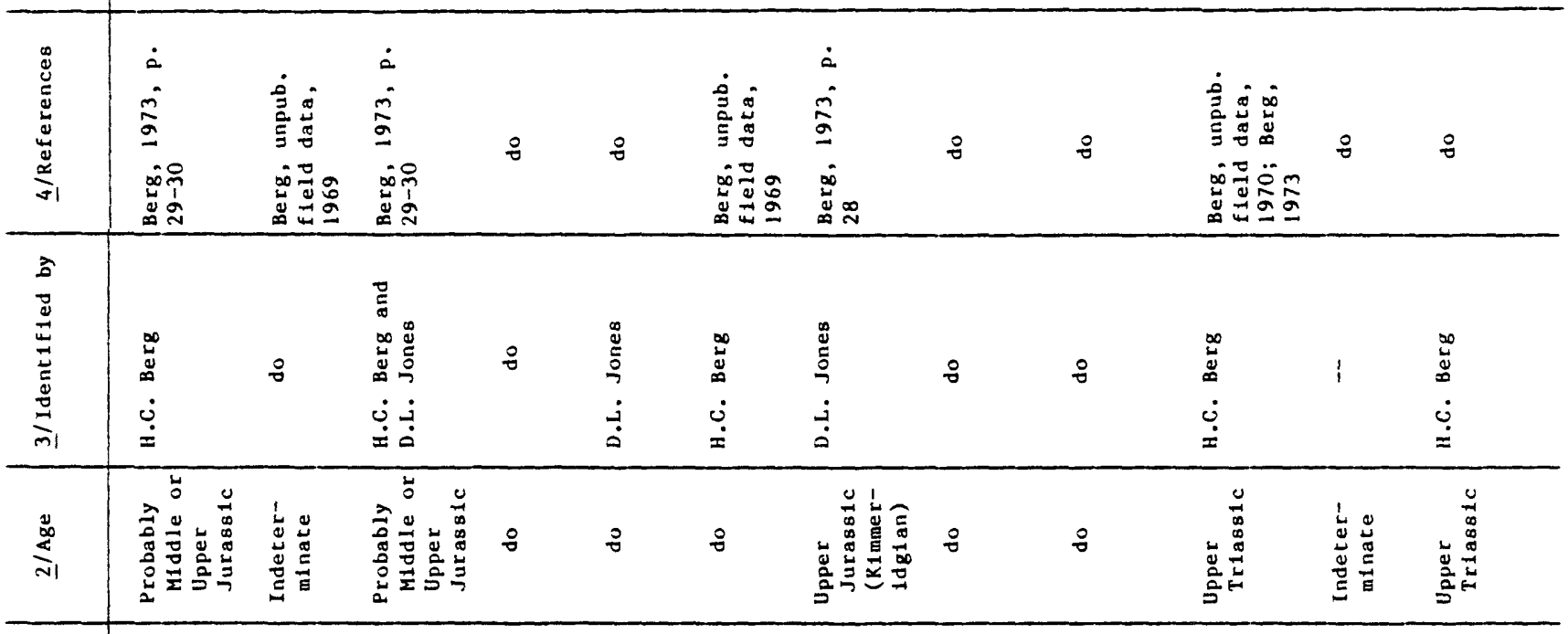

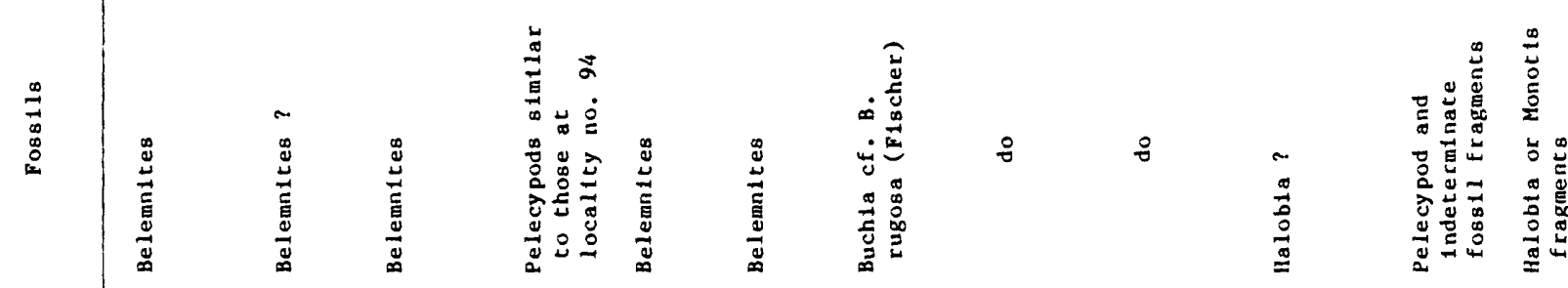

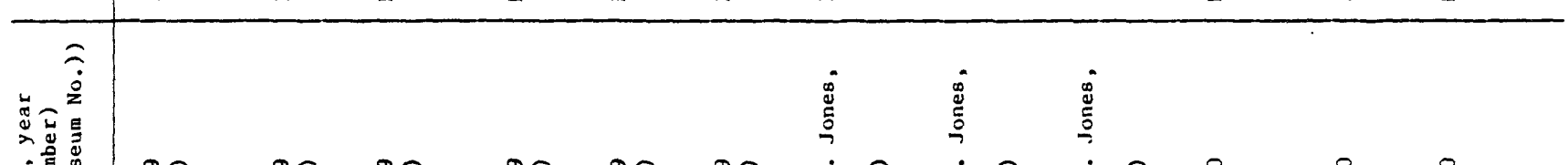

प)

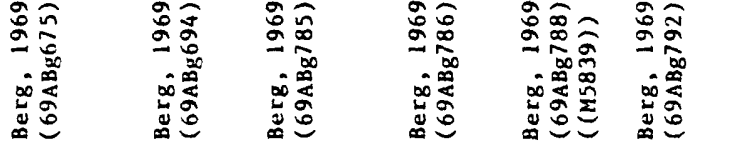

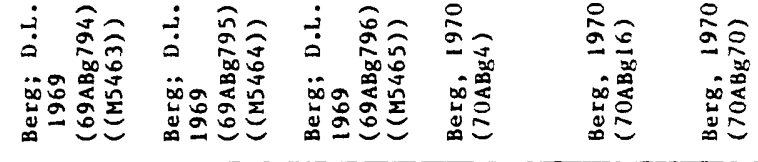

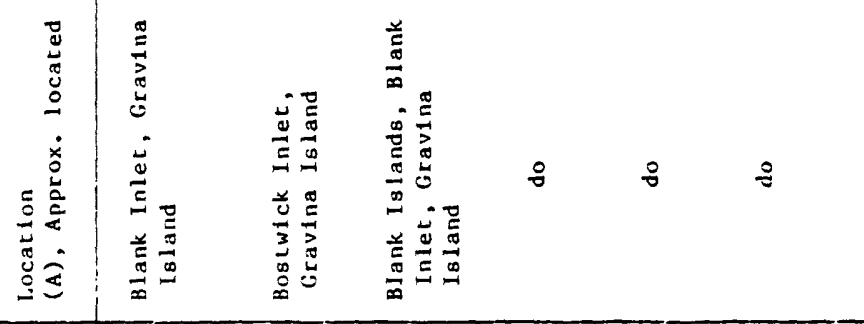

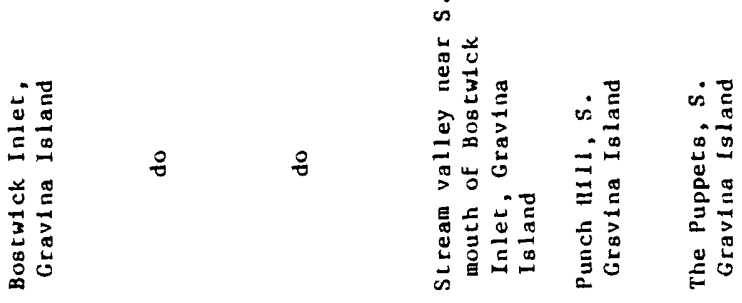

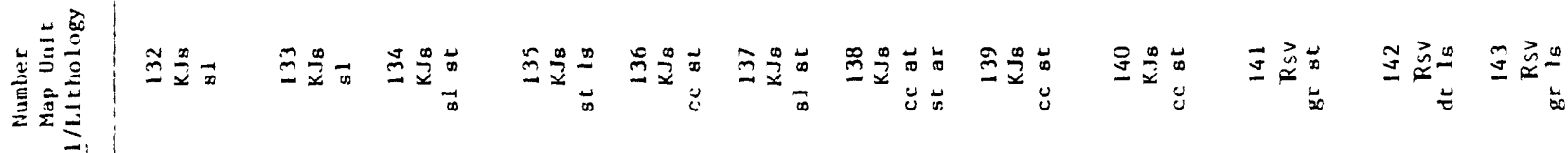




\begin{tabular}{|c|c|c|c|c|c|c|c|c|c|}
\hline 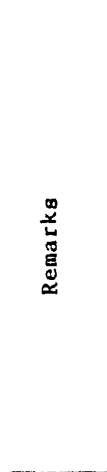 & & & & & 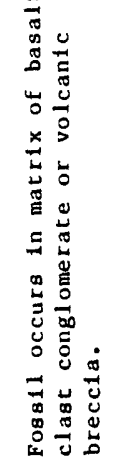 & 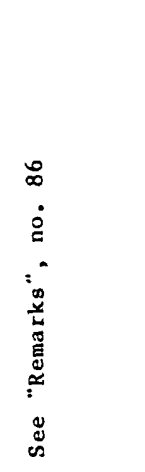 & $\stackrel{8}{8}$ & & 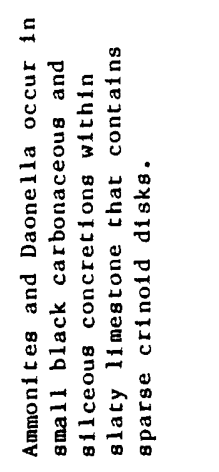 \\
\hline 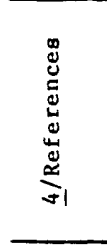 & 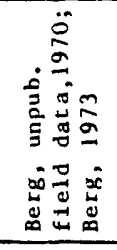 & $\stackrel{8}{7}$ & $\stackrel{\circ}{\square}$ & $\stackrel{8}{7}$ & $\stackrel{8}{7}$ & 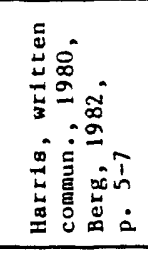 & 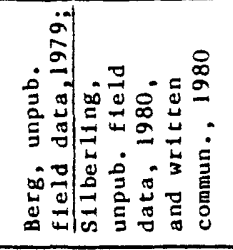 & 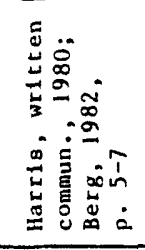 & 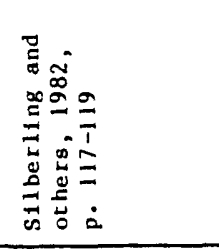 \\
\hline 会 & 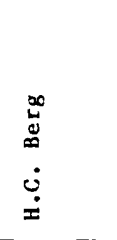 & $\stackrel{\circ}{v}$ & $\stackrel{8}{7}$ & $\stackrel{\circ}{\circ}$ & $\stackrel{\circ}{\square}$ & 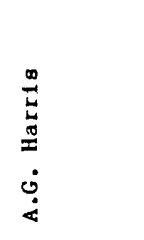 & 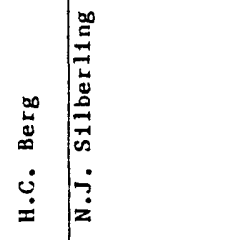 & $\begin{array}{l}\stackrel{\infty}{\underbrace{0}_{0}} \\
\stackrel{5}{=} \\
\dot{0} \\
\dot{\alpha}\end{array}$ & 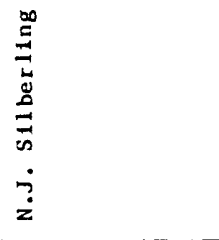 \\
\hline$\stackrel{\text { जा }}{\stackrel{2}{a}}$ & 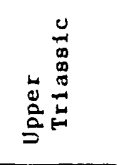 & $\stackrel{\circ}{7}$ & $\because$ & $\stackrel{\circ}{7}$ & 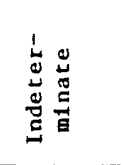 & 意 & 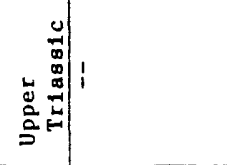 & 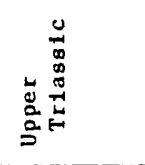 & 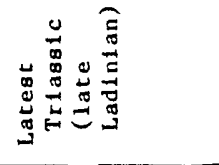 \\
\hline 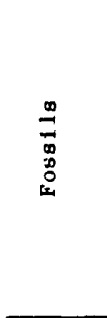 & 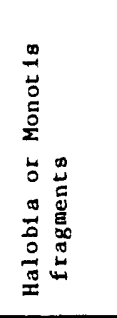 & 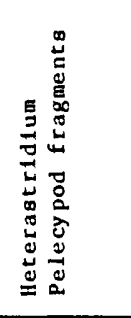 & 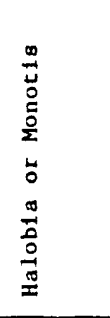 & 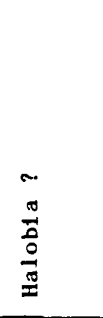 & 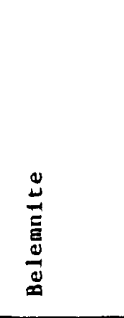 & 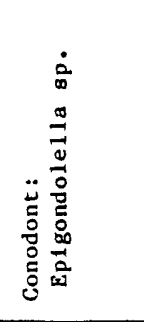 & 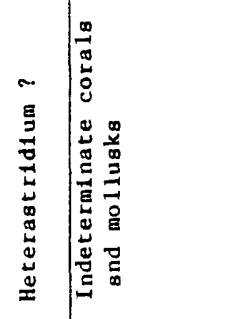 & 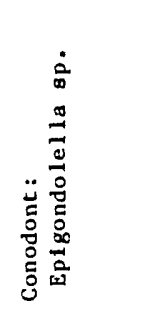 & 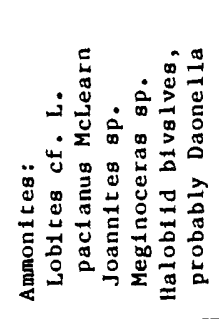 \\
\hline 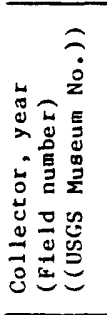 & 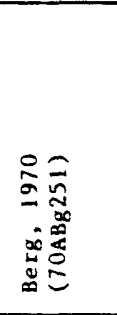 & 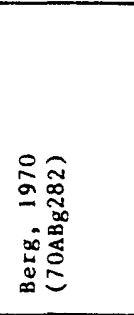 & 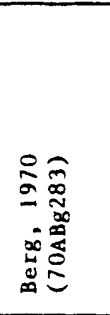 & 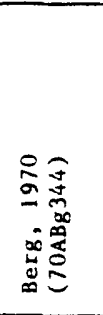 & 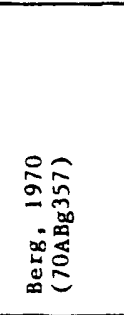 & 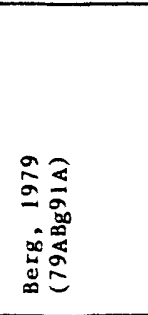 & 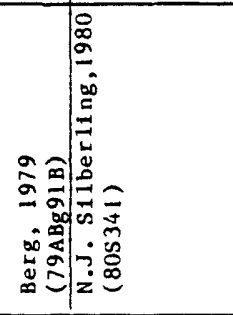 & 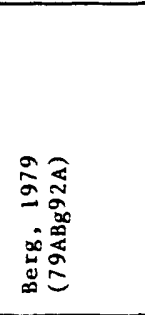 & 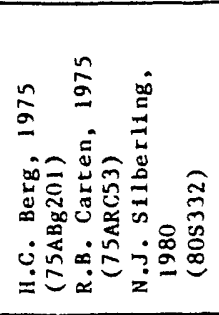 \\
\hline 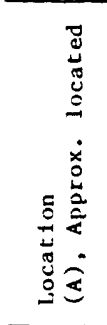 & 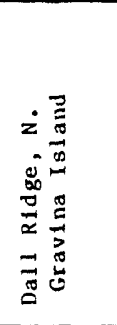 & 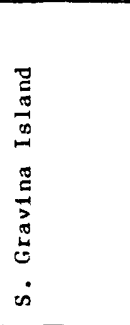 & $\stackrel{8}{7}$ & 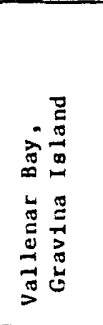 & 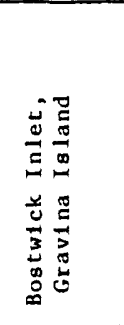 & 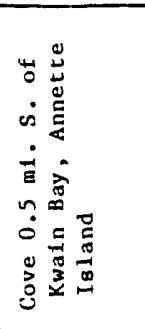 & 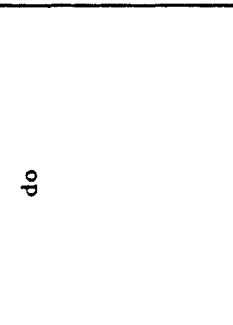 & 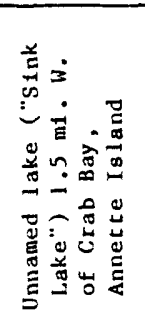 & 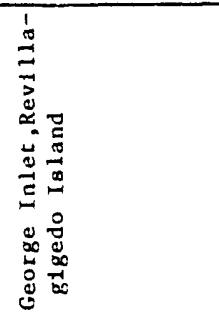 \\
\hline 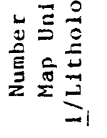 & 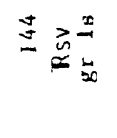 & 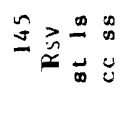 & 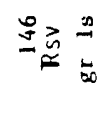 & $\Xi \overrightarrow{\tilde{\aleph}}^{\infty}$ & $\stackrel{\infty}{=} \underset{\approx}{\approx} 0$ & $\stackrel{\sigma}{=} \ddot{\check{c}}$ & 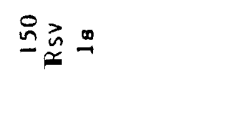 & 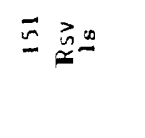 & 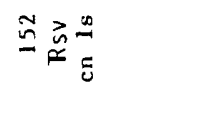 \\
\hline
\end{tabular}




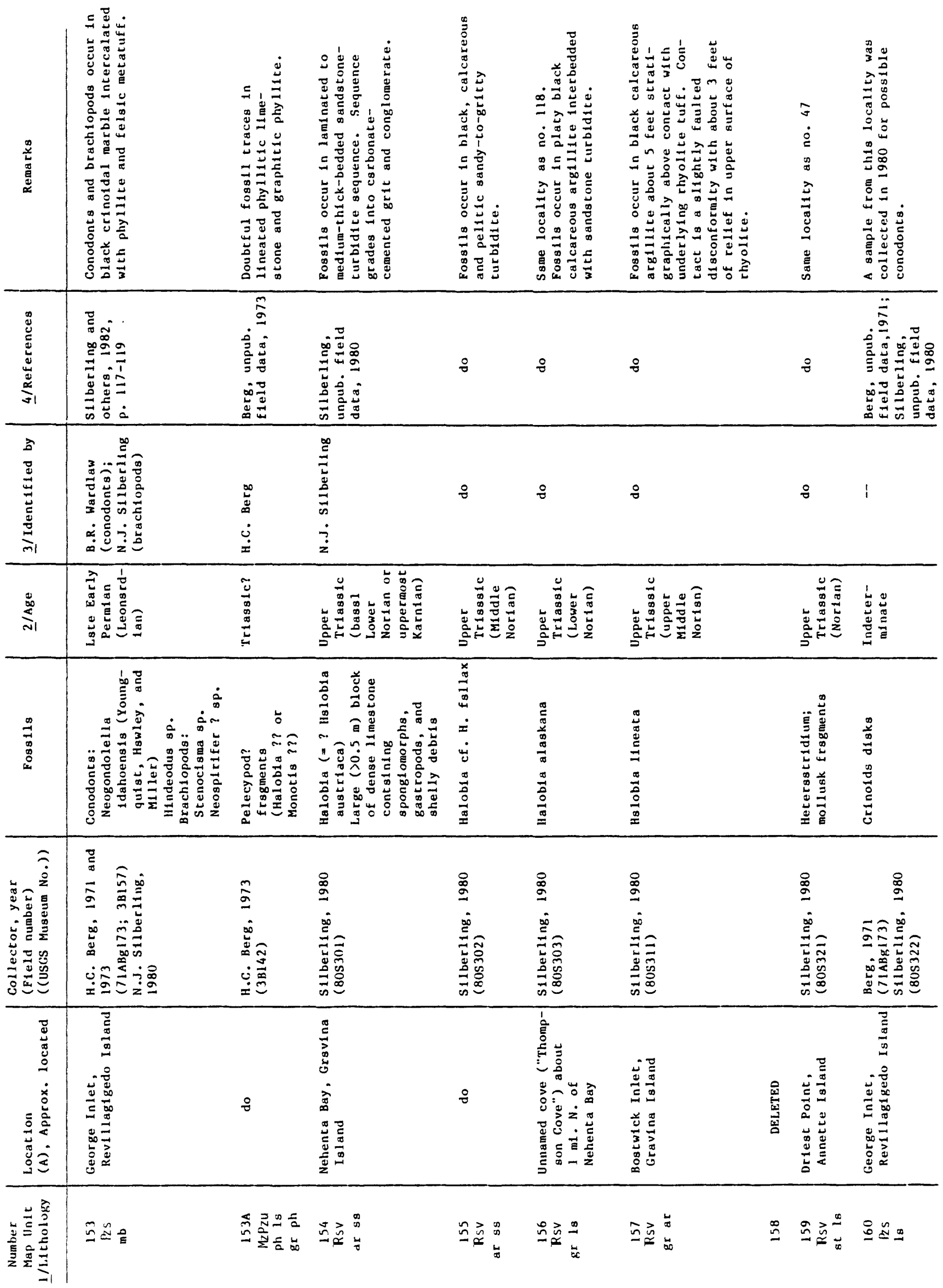




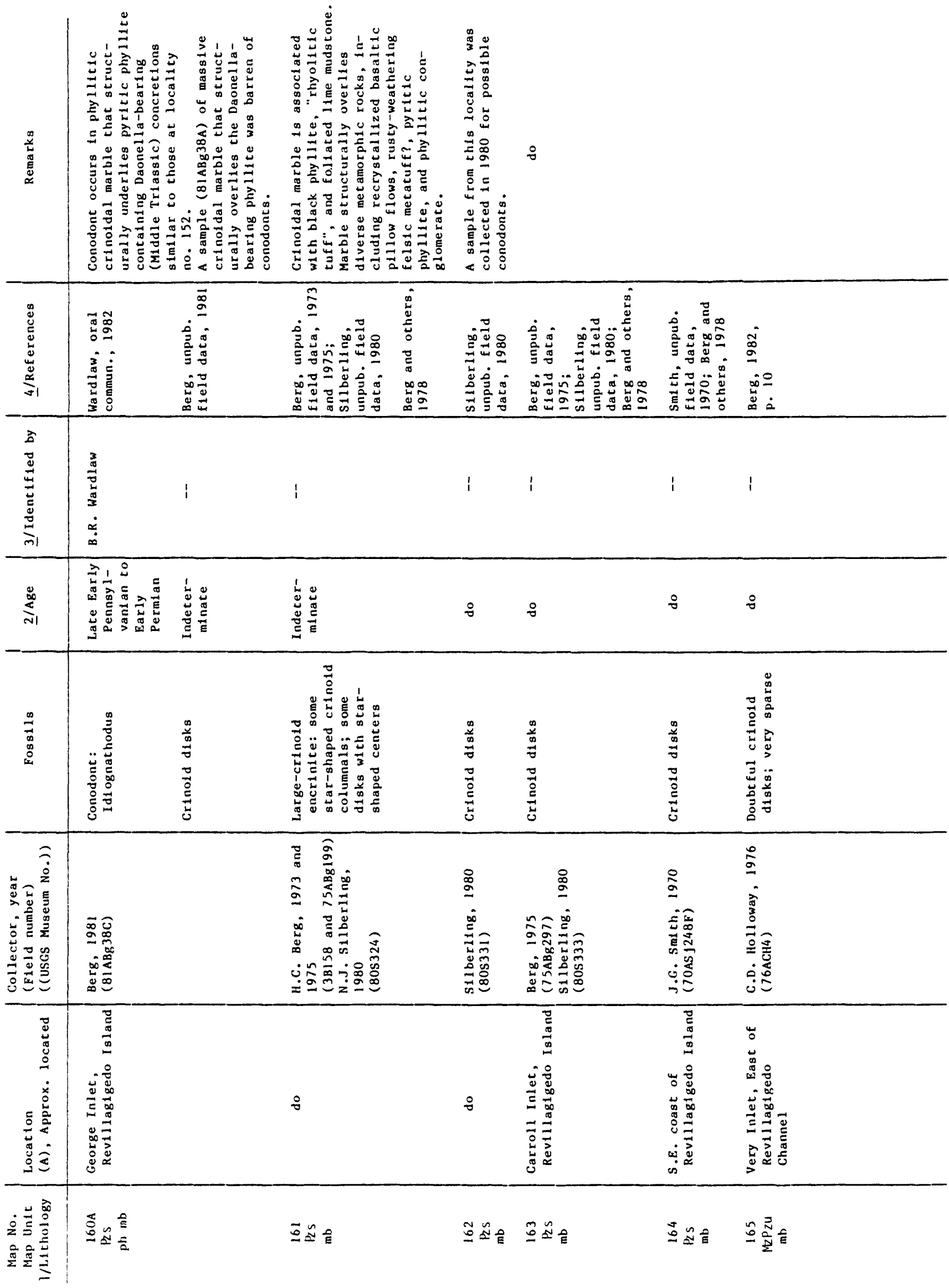




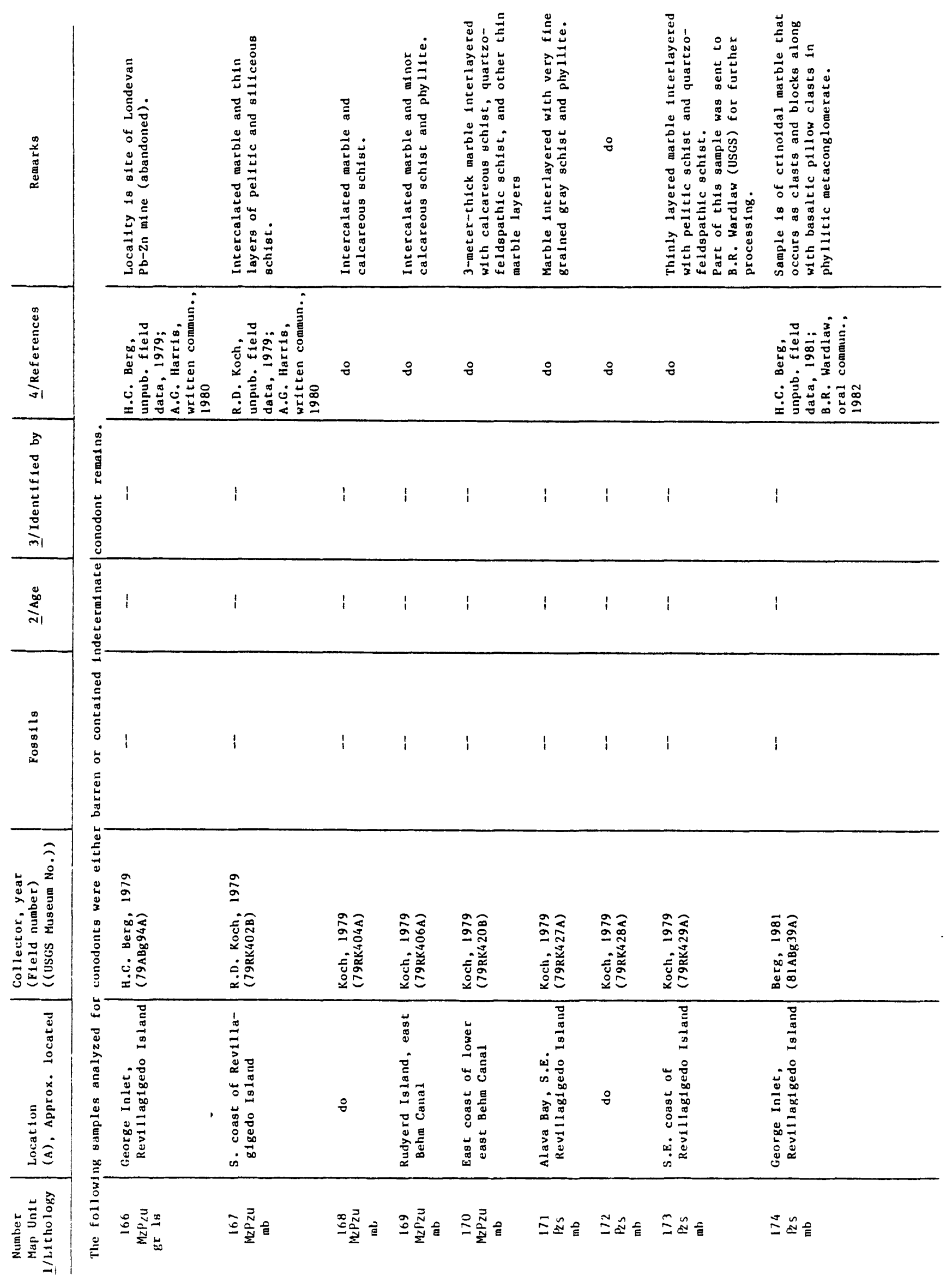

